\title{
Chronique du Colloque International « Port-Royal et la philosophie »
}

Catane, 8-10 novembre 2010

\section{Maria Vita Romeo}

\section{OpenEdition}

\section{Journals}

Édition électronique

URL : http://journals.openedition.org/ccibp/295

DOI : $10.4000 /$ ccibp. 295

ISSN : 2493-7460

\section{Éditeur}

Centre international Blaise Pascal

Édition imprimée

Date de publication : 1 décembre 2011

Pagination : 14-22

ISBN : 978-2-84516-551-9

ISSN : 0249-6674

\section{Référence électronique}

Maria Vita Romeo, « Chronique du Colloque International « Port-Royal et la philosophie » », Courrier du Centre international Blaise Pascal [En ligne], 33 | 2011, mis en ligne le 19 novembre 2015, consulté le 11 novembre 2020. URL : http://journals.openedition.org/ccibp/295; DOI : https://doi.org/10.4000/ccibp. 295

Ce document a été généré automatiquement le 11 novembre 2020.

Centre international Blaise Pascal 


\title{
Chronique du Colloque International « Port-Royal et la philosophie»
}

Catane, 8-10 novembre 2010

\author{
Maria Vita Romeo
}

Port-Royal représente une expérience historique de haut niveau, qui dépasse et les frontières de la France et les frontières du XVII ${ }^{e}$ siècle. Faire référence à Port-Royal, comme l'on sait, signifie reporter à la mémoire de grands événements historiques de correction morale et religieuse dans le cadre de l'Église, des expériences profondes et sincères de spiritualité, des styles de vie fondés sur la pauvreté, la prière et la réflexion, des batailles passionnées contre le laxisme éthique, des collisions violentes entre jansénistes et jésuites. Et quand, par décret de Louis XIV, l'abbaye de Port-Royal sera rasée au sol et ses religieuses contemplatives seront "déportées », alors restera intact pour toujours le trésor de multiples expériences culturelles et religieuses. Et l'historien qui interroge ces décombres entend l'écho de la passion philosophique pour Descartes, pour saint Augustin, pour Platon ; et il écoute les voix puissantes de Pascal et de Racine, $\mathrm{du}$ Grand Arnauld et de Nicole; voix qui exaltent une forme moderne de faire de la philosophie ensemble, qui renouvelle presque l'Académie de Platon par le sumphilosophein dans la communauté d'idées, de foi et d'étude; voix qui racontent l'événement éternel du dualisme entre l'amour de Dieu et l'amour de l'homme, entre la vérité religieuse et la vérité scientifique, entre liberté et nécessité, entre beauté et vérité, entre fini et infini.

2 Mais, pour parler avec rigueur, peut-on déterminer et définir une «philosophie de Port-Royal » ? Il n'est pas facile de donner une réponse affirmative. Nous pouvons dire, cependant, que des philosophes et des philosophies animèrent la remarquable vie culturelle et spirituelle de Port-Royal avec un horizon spéculatif qui comprend l'Antiquité, les temps modernes, l'ardente spiritualité chrétienne et la mentalité laïque et scientifique. Sur ce microcosme philosophique, le Colloque International sur « PortRoyal et la philosophie » a été organisé (Catane, 8, 9 et 10 novembre 2010) dans 
l'amphithéâtre du Monastère des Bénédictins, siège de la Faculté de Lettres et Philosophie de l'Université de Catane.

3 Ce Colloque - inspiré par deux maîtres des études pascaliennes, Jean Mesnard et Philippe Sellier - a été organisé par le Centre International Blaise Pascal de ClermontFerrand, dirigé depuis longtemps par Dominique Descotes; par la Société des Amis de Port-Royal de Paris actuellement dirigé par Jean Lesaulnier et par la Cattedra di Filosofia Morale de l'Université de Catane, dirigé par Giuseppe Pezzino. Trois Centres dont la collaboration fait ressortir la volonté commune de réaliser une stratégie de politique culturelle qui recueille autour des thématiques $d u X V I I^{e}$ siècle des énergies intellectuelles mondiales.

4 À l'ouverture des travaux Giuseppe Pezzino a souligné l'articulation et la structure équilibrée d'un programme de travail qui, en partant de la conviction qu'on ne peut pas parler d'une "philosophie de Port-Royal ", se propose d'examiner les philosophes qui ont animé la vie intellectuelle de Port-Royal et, surtout, les philosophies qui ont traversé ces lieux de prière et de spiritualité.

5 Ensuite, Laurence Devillairs et Patricia Touboul ont exposé l'objectif du Colloque: parcourir un itinéraire qui porte son attention sur la philosophie ancienne, afin de mettre à nu les racines philosophiques de Port-Royal, pour passer, successivement, à la philosophie moderne, animée par l'esprit cartésien et imprégnée d'augustinisme, et à la fin retrouver les échos des sujets port-royalistes dans l'histoire moderne et contemporaine.

6 La première session du Colloque, dédiée à Port-Royal et la philosophie ancienne, s'ouvre avec la communication de Jean Lesaulnier (Société des Amis de Port-Royal) qui conduit la nombreuse assistance dans le microcosme de l'enquête philosophique port-royaliste, et précisément dans le château de Vaumurier qui, à côté du salon de la marquise de Sablé, est un des lieux « élevés » où les penseurs de Port-Royal débattaient de littérature, de philosophie, de théologie et de science, en se faisant ainsi promoteurs des nouveautés cartésiennes, même s'il restent fidèles à saint Augustin. Dans la communication suivante, Hélène Michon (Université de Tours) analyse les vicissitudes de la notion de plaisir à Port-Royal, en prenant comme point de comparaison François de Sales et Bossuet, dans la tentative de saisir l'originalité de Pascal pour lequel le plaisir rappelle exclusivement le plaisir des sens, en l'associant, ainsi, à l'idée de concupiscence augustinienne, identifiée avec le péché.

7 L'intéressante communication de Philippe Sellier (Université Paris-Sorbonne), fait référence aux racines de la pensée ancienne pour souligner le rapport entre la philosophie et Port-Royal. Tout en indiquant la connaissance limitée des textes platoniques en langue originale à Port-Royal, Sellier souligne l'influence - même si à travers saint Augustin - exercée par le grand Athénien entre les murs de l'abbaye française. Philippe Sellier aborde le sujet du platonisme à Port-Royal sur deux axes : la théologie platonique, qui a été objet d'études depuis l'époque de Jansénius et de SaintCyran; et le platonisme esthétique, qui s'est répandu seulement en quelques-unes des personnalités de la seconde génération de Port-Royal, et notamment en Pierre Nicole.

8 La seconde session de la première journée du Colloque a été dédiée à «La philosophie moderne: cartésianisme et anti-cartésianisme». Ici nous trouvons la relation intéressante de Laurence Devillairs (Éditions du Seuil, Paris) qui aborde, à l'intérieur de la philosophie moderne, le problème de l'homme imago Dei, en soulignant de façon originale la relation entre Descartes et saint Augustin sur le rapport entre l'homme fini 
et Dieu infini. Ainsi, sur la ligne de démarcation du cartésianisme et de l'anticartésianisme, Devillairs affirme que, selon Descartes, grâce à l'idée d'infini, on parvient à la connaissance et à l'adoration de Dieu. De telle manière, Descartes, sur le sujet de l'homme comme imago Dei, résulte «plus augustinien » que Pascal. Un Pascal qui, selon Devillairs, opérerait un effacement de l'image de Dieu dans l'homme, en l'éloignant du milieu augustinien.

9 Avec la communication de Domenico Bosco (Université de Chieti-Pescara), l'antinomie cartésianisme/anti-cartésianisme se dilate dans la plus générale antinomie de philosophie/antiphilosophie. Et en partant du Liber procmialis du tome II de l' Augustinus, où on regarde avec suspicion une philosophie qui, en ignorant l'Écriture sainte, va se fonder sur l'humana ratio pour affronter les problèmes de la vie spirituelle. De plus, Domenico Bosco fait remarquer comme-au-delà de la même position d'ensemble de Port-Royal en ce qui concerne le monde - au cours du XVII siècle la philosophie réalise un procès d'« auto-compréhension » qui aboutit à la considération critique de la foi religieuse et de la philosophie même. Et, en ce cas, l'orientation toute spirituelle du «premier Port-Royal» cède le pas aux nouvelles exigences qui mèneraient Port-Royal à se mesurer avec le monde, en abordant les vérités religieuses avec des modalités intéressées aux provocations critiques du temps.

10 En outre, Bosco réfléchit sur l'importance de la philosophie à Port-Royal, aussi grâce à l'analyse d'une œuvre pascalienne de nature philosophique souvent restée dans l'ombre : L'Entretien avec M. de Sacy. Enfin, la communication de Bosco observe que, audelà des choix des Messieurs de Port-Royal, il y a une philosophie qui se met au service de la vérité, qui doit être cherchée avec méthode, comme le montre la Logique ou l'art de penser.

11 La première session de la deuxième journée du colloque est dédiée aux figures des philosophes; cette session est ouverte par la communication de Denis Moreau (Université de Nantes) sur A. Arnauld, dont les textes de "philosophie» constituent une partie restreinte de l'œuvre immense d'une des plus grandes personnalités de PortRoyal. À ce propos, Moreau nous offre un bilan précieux en soulignant cinq points essentiels : la philosophie d'Arnauld, qui est l'expression la plus nette de son intérêt pour la philosophie de Descartes, ses idées, le jansénisme, le cartésianisme et le problème des vérités éternelles.

Dans cette même sphère d'intérêt pour les figures des philosophes, Laurent Thirouin (Université de Lyon) s'interroge sur la possibilité d'attribuer de la valeur philosophique à une partie de l'œuvre de Pierre Nicole. Ainsi, en partant de la réflexion morale du philosophe de Port-Royal, Thirouin soutient remarquablement que Nicole philosophe moraliste est indispensable pour libérer Port-Royal de beaucoup de préjugés qui se sont accumulés au cours des années.

13 Jean Mesnard (Université Paris-Sorbonne, membre de l'Institut) concentre son attention sur l'importance de la philosophie à Port-Royal, et sur l'existence d'une philosophie pascalienne à Port-Royal. Sans aucun doute, au XVII ${ }^{e}$ siècle, autour de l'abbaye de Port-Royal, exceptionnellement, se constitue un groupe intellectuel vif, avec Pascal comme membre le plus éminent, qui ne peut pas manquer de réfléchir sur les idées de vérité et de bien en présence soit du scepticisme, qui se sert du doute pour blesser mortellement toutes les certitudes, soit de la science moderne avec ses nouveaux modèles de certitude et de sûreté. Bref, comme Jean Mesnard nous l'a magistralement indiqué, à Port-Royal on sent la présence de Descartes 
indubitablement, mais on sent également la présence de Pascal qui agrandit la méthode géométrique à tel point qu'il préfigure une Mathesis universalis, qui ne dédaigne pas l'enquête philosophique et qui s'arrête seulement devant la sphère de la foi et de la Révélation.

La seconde session du 9 novembre, dédiée à la Philosophie et à la critique de la philosophie, s'ouvre avec la communication d'Alberto Peratoner (Studium Generale Marcianum, Venise) qui, en recherchant sur les rapports entre foi et raison, met en contraste les forces imposantes d'une spiritualité rigoureuse qui vise au transcendant et qui regarde avec suspicion et condamnation toute activité profane et mondaine, comme la philosophie ou la science.

nous avons la communication de Maria Vita Romeo (Université de Catane) qui oriente sa réflexion sur la valeur morale de la Logique de Port-Royal, une œuvre qui ne se contente pas d'être une "logique sèche", mais qui comprend en elle-même "une quantité de choses pertinentes " pour toutes les sciences qui, d'une certaine manière, participent à une vraie formation du jugement. C'est pour cela que les auteurs de la Logique, parmi les quatre opérations principales de l'esprit (concevoir, juger, raisonner et ordonner), réservent la plus haute importance au jugement, car de la qualité du jugement dépend «l'exactitude de la raison », dont l'utilisation est "utile dans toutes les parties et dans tous les emplois de la vie». Donc, selon Maria Vita Romeo, le principal but de la Logique, suivant l'exemple de saint Augustin, consiste à former l'homme, qui doit apprendre à se connaître, pour devenir meilleur. En réalisant une espèce de synthèse entre Descartes et Pascal, les auteurs de la Logique soulignent que l'homme doit apprendre à bien penser, puisque penser bien signifie aussi et surtout agir bien.

16 La troisième session de la deuxième journée est dédiée au rapport Science et Logique à Port-Royal. Elle est ouverte par la communication sur La philosophie des mathématiques à Port-Royal de Dominique Descotes (Université de Clermont-Ferrand). Sur cette base de réflexion, Descotes se propose de comprendre comment Pascal conçoit les mathématiques à partir de la manière dont il les pratique. À cette fin, en comparant de manière magistrale les mathématiques de Pascal avec les mathématiques de Port-Royal, et en prenant en particulier en considération l'idée d'ordre comme elle est recherchée en Dettonville, Descotes arrive à la conclusion que pour Pascal l'arithmétique précède et fonde la géométrie.

17 Enfin, on passe à la communication d'Hubert Aupetit (Société des Amis de Port-Royal) sur L'Infini des philosophes: la démystification pascalienne. Aupetit aborde la manière avec laquelle Pascal se sert de la notion d'infini comme élément déterminant dans une enquête anthropologique qui commence de L'Esprit géométrique pour continuer dans les Provinciales et dans les Pensées. Si pour Descartes l'infini caractérise Dieu, pour Pascal, selon Aupetit, devient un attribut de la pensée humaine, la marque empirique de ce qui pour l'homme est inaccessible à la raison pure. C'est la réflexion sur l'infini qui pousse Pascal à quitter le champ philosophique pour investir un champ original, guidé par l'« esprit géométrique ». La notion d'infini fait comprendre au géomètre qu'aucune pensée, même la plus mathématiquement formulée, n'est assurée dans ses fondements : dès lors, le seul accès de la raison à la vérité est indirect, et relève du raisonnement par l'absurde.

18 La troisième et dernière journée du colloque dédiée à La postérité de la philosophie à PortRoyal s'ouvre avec la relation de Martine Pécharman (EHESS - CNRS) qui nous invite à 
réfléchir sur les échos de la Logique dans l'Essay Concerning Human Understanding de Locke. À cet Essay, l'on doit véritablement "la direction de l'esprit humain dans la recherche de la connaissance», parce qu'il marque la fondation d'une "nouvelle logique ", dont en tout cas la Logique de Port-Royal se fait porteuse, en contrevenant à la Logique aristotélicienne.

Et encore la Logique de Port-Royal est au centre de la relation intéressante d'Hélène Bouchilloux (Université de Nancy). Dans la Logique, affirme H. Bouchilloux, Arnauld et Nicole adoptent l'innéisme cartésien contre Hobbes, reprochant à celui-ci d'avoir une philosophie des mots plutôt que des choses. Les auteurs de la Logique se rapportent à Descartes et à la manière dont il pense la connaissance de soi, la connaissance de Dieu et la connaissance mathématique sans faire les comptes avec Pascal qui, en revanche, récuse cette façon de penser la connaissance de soi, la connaissance de Dieu et la connaissance mathématique.

Ensuite, la communication de Patricia Touboul (Université de Montpellier) part de l'idée d'«acrasie» ou "faiblesse» de la volonté, pour aborder le problème philosophique et moral de Pierre Nicole. Le terme «acrasie »-souligne Touboul - ne correspond à aucun fait stricto sensu, mais rend possible une interprétation permettant de distinguer des actions sur le plan pratique, selon leur degré de conformité à des règles, sans prétendre d'apporter une justification ontologique. Dans cette optique, la question de la réalité du phénomène est examinée brièvement afin de délimiter le terrain sur lequel se situe l'analyse de Nicole ainsi que la proximité qu'elle entretient avec les conclusions des débatteurs contemporains. En vérité, le problème de l'acrasie a été initialement abordé par les philosophes de l'antiquité, et successivement par les Pères de l'Église et les moralistes français du Grand Siècle. De toute façon, dans l'Essai de la faiblesse de l'homme, Pierre Nicole conteste l'usage même de l'idée d'acrasie, en relevant essentiellement à l'origine d'une problématique morale-il s'agit de déterminer si le mal était commis volontairement - une question qui fait aujourd'hui l'objet d'un réexamen inhérent à l'éclaircissement de problèmes sur la logique de l'action en sens large.

21 La fermeture du colloque est confiée à Giuseppe Pezzino (Université de Catane) qui tire les conclusions des trois journées de travail intellectuel fervent et fécond, en parcourant un chemin marqué par seize communications d'une grande valeur scientifique.

22 Après avoir pris en considération les points essentiels du programme et des communications, et après avoir communiqué au public qu'en 2011 les actes du colloque seront publiés dans les Chroniques de Port-Royal, Giuseppe Pezzino fait le bilan d'un colloque international qui a offert non seulement de considérables occasions d'enrichissement intellectuel, de sollicitation culturelle et de chaleur humaine, mais il a aussi fait place à l'avenir, en sollicitant de nouvelles questions et en préparant de nouvelles énergies intellectuelles pour de nouveaux itinéraires scientifiques.

Mercredi 9 dans l'après-midi, les auditeurs et les intervenants ont effectué une agréable excursion au Taormina, où le soir ils ont dîné dans un climat de sympathie et d'amitié. Dans cette occasion, tous les congressistes ont voulu honorer Jean Mesnard en lui offrant une plaque d'argent en signe de dévotion profonde et de vive gratitude. 